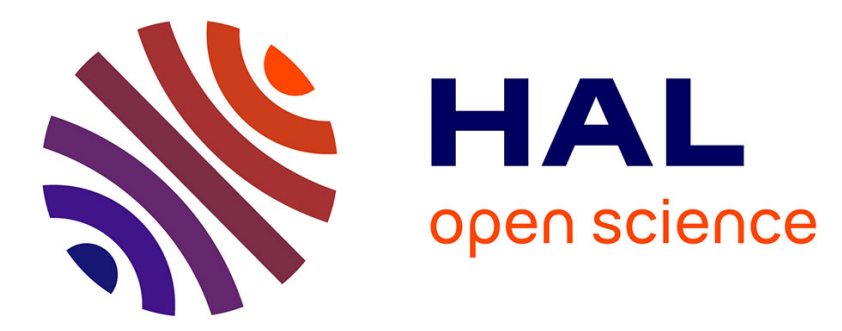

\title{
Disentangling subgroups of participants recruiting shared as well as different brain regions for the execution of the verb generation task: A data-driven fMRI study
} Leonardo Cerliani, Rajat Mani Thomas, Domenico Aquino, Valeria Contarino, Alberto Bizzi

\section{To cite this version:}

Leonardo Cerliani, Rajat Mani Thomas, Domenico Aquino, Valeria Contarino, Alberto Bizzi. Disentangling subgroups of participants recruiting shared as well as different brain regions for the execution of the verb generation task: A data-driven fMRI study. Cortex, 2016, 86, pp.247-259. 10.1016/j.cortex.2016.11.017 . inserm-01433145

\section{HAL Id: inserm-01433145 https://www.hal.inserm.fr/inserm-01433145}

Submitted on 12 Jan 2017

HAL is a multi-disciplinary open access archive for the deposit and dissemination of scientific research documents, whether they are published or not. The documents may come from teaching and research institutions in France or abroad, or from public or private research centers.
L'archive ouverte pluridisciplinaire HAL, est destinée au dépôt et à la diffusion de documents scientifiques de niveau recherche, publiés ou non, émanant des établissements d'enseignement et de recherche français ou étrangers, des laboratoires publics ou privés. 


\title{
Disentangling subgroups of participants recruiting shared as well as different brain regions for the execution of the verb generation task: a data-driven fMRI study.
}

Leonardo Cerliani ${ }^{1}, 2$, Rajat Mani Thomas 3 , Domenico Aquino4, Valeria Contarino ${ }^{4}$ and Alberto Bizzi4

${ }^{1}$ Frontlab, Brain Connectivity and Behaviour, Institut du Cerveau et de la Moelle épinière (ICM), Paris, France.

2Inserm U1127, GH Pitié-Salpêtrière, 75013, Paris, France.

${ }^{3}$ Department of Psychiatry, Academic Medical Center, University of Amsterdam, Amsterdam, The Netherlands

${ }^{4}$ Neuroradiology Department, IRCCS Foundation Neurological Institute Carlo Besta, Via Celoria 11, 20133 Milan, Italy

Last revision - Accepted for publication in Cortex, January 2017

http://www.sciencedirect.com/science/article/pii/S0010945216303434

\begin{abstract}
The spatial pattern of task-related brain activity in fMRI studies might be expected to change according to several variables such as handedness and age. However this spatial heterogeneity might also be due to other unmodeled sources of inter-subject variability. Since group-level results reflect patterns of task-evoked brain activity common to most of the subjects in the sample, they could conceal the presence of subgroups recruiting other brain regions beyond the common pattern. To deal with these issues, data-driven methods can be used to detect the presence of sources of inter-subject variability that might be hard to identify and therefore model a priori. Here we assess the potential of Independent Component Analysis (ICA) to detect the presence of unexpected subgroups of participants. To this end, we acquired task-evoked fMRI data on 45 healthy adults using the verb generation task, in which participants are visually presented with the noun of an object of everyday use, and asked to covertly generate a verb describing the corresponding action. As expected, the task elicited activity in a temporo-parietofrontal network typically found in previous verb generation experiments. We then quantified the contribution of every subject to nine task-related spatio-temporal processes identified by ICA. A cluster analysis of this quantity yielded three subgroups of participants. Differences between the three identified subgroups were distributed in left and right prefrontal, posterior parietal and extrastriate occipital regions. These results could not be explained by differences in sex, age or handedness across the participants. Furthermore, some regions where a significant difference was found between subgroups were not present in the group-level pattern of task-related activity. We discuss the potential application of this approach for characterizing brain activity in different subgroups of patients with neuropsychiatric or neurological conditions.
\end{abstract}

Keywords: ICA; fMRI; verb generation; inter-subject variability 


\section{Introduction}

Many fMRI studies aim at localizing brain regions whose activity is enhanced while performing a given cognitive task. Usually this involves estimating how consistent is the effect of the task on the regional haemodynamic response across participants, by testing the difference from zero in the group-average parameter estimates at each brain voxel (Holmes and Friston, 1998). This random effect analysis, which allows generalizing the results beyond the considered sample, relies on the assumption that participants have been drawn from the same homogeneous population (Kherif, 2003). Inter alia, this means assuming that most or all of the participants performed the task using the same set of brain regions, while task-related activity detected elsewhere in the brain of single participants - or possibly characterizing only a subgroup of participants - will be discarded as a confound related to random noise (Noppeney et al., 2004). Rarely, however, this assumption is made the object of a verification.

The issue of how to deal with spatial heterogeneity in task-related brain activity between subjects is not only methodologically relevant (Price and Friston, 2002; Friston and Price, 2003): several studies have shown how different sources of inter-subject variability can lead to differences in the location of the brain regions recruited during the performance of the same task across subjects. For instance, Kherif and colleagues (Kherif et al., 2009) analyzed data from a reading aloud task in a heterogeneous sample of 76 neurotypical participants featuring different demographic characteristics (handedness, sex, age). This study aimed at extracting, in a data-driven fashion, the principal trajectories of demographic variability associated with the spatial distribution of activation patterns across participants. The four identified subgroups did not differ in terms of handedness and sex, however younger (adolescent) participants showed stronger left-lateralized activity. The other three groups showed an independent contribution above the common pattern in the ventrolateral premotor and extrastriate visual cortex bilaterally, as well as in the left posterior cingulate region. Remarkably however the latter subgroups did not differ from each other in terms of the recorded demographic variables. The latter result highlights the fact that subgroups characterized by different patterns of brain activity might reflect sources of variability that can be difficult to define a priori. For instance, in a study focused on reading familiar words (Seghier et al., 2008) on righthanded subjects, subgroups of participants showed different levels of task-related activity in either left posterior occipito-temporal and right inferior parietal regions, or in left anterior occipito-temporal and left ventral inferior frontal regions, respectively. This variability could not be explained in terms of gender, age or stimulus differences, while it was associated with the speed of reading irregular words versus pseudowords. Finally, the presence of commonalities and differences in the pattern of brain activity associated with a task could potentially suggest the implementation of different cognitive strategies in different subgroups (Price and Friston, 2002), as elaborated in a study (Noppeney et al., 2006) involving the estimation of the weight of an object, presented visually and primed via auditory stimuli. Different levels of activity in occipital and posterior temporal regions bilaterally, as well as in the right inferior posterior parietal cortex, between the two identified subgroups, hinted at the possibility that in order to perform the task some participants relied more on visual information, while some other relied more on auditory cues. This evidence supports the idea that in a sample of participants performing the same task during an fMRI session, subgroups characterized by different phenotypical features and/or using different cognitive strategies can display variability in the location of the brain regions recruited for that task. Failing to model the presence of these subgroups can have profound effects on the group-level results and on their interpretation; if different subgroups of participants perform the same task using both common and different brain regions, the locations where consistent activity is detected in the whole sample will potentially represent only a subset of the brain regions which are necessary and sufficient to perform that task (Noppeney et al., 2006).

In summary, both theoretical and methodological considerations highlight the importance of probing, rather than assuming a priori, the homogeneity of the sample with respect to the brain regions recruited to perform the same task in the scanner. This represents a hard task since subgroups of participants featuring different patterns of brain activity might be characterized by unexpected, and therefore unmodeled sources of inter-subject variability. In this situation, applying data-driven methods to subject-level profiles of brain activity can provide a valuable strategy to identify the presence of unexpected subgroups. This strategy was indeed adopted by a few aforementioned studies (Noppeney et al., 2006; Kherif et al., 2009), which analyzed the brain maps of voxel wise 
differences (i.e. error variance) between subject-specific and group-level parameter estimates of task-related activity. Different subgroups of participants were detected on the basis of their differential expression of the principal components of these maps, quantifying the error variance with respect to the group mean. One potential issue in this approach is that some part of the error variance might reflect noise due to motion, physiological artifacts, scanner drifts, rather than task-related neuronal activity (Beckmann, 2012). The ability to regress out these sources of noise would increase the confidence in the fact that differences in the pattern of activations across subgroups are driven by differences in task-related brain activity.

In the present work we consider how the detection of subgroups of participants can be carried out on the basis of spatio-temporal patterns of brain activity, that is spatial maps whose temporal descriptor is associated with the execution of the task, and in which the presence of task-unrelated noise has been regressed out prior to the analysis. To this aim, we process fMRI data obtained during a verb generation task (Bizzi et al., 2008), using the Tensorial extension of Probabilistic Independent Component Analysis (T-PICA) (Beckmann and Smith, 2005). In recent years, ICA has become a popular method to extract spatial maps and their associated time courses in resting-state fMRI data. These maps resemble functional networks recruited during actual task-based experiments (Smith et al., 2009), and have proven advantageous to un-mix signal associated with noise from that related to brain activity (Beckmann, 2012). Since ICA is blind to the conditions in which the fMRI data were acquired, the same procedure can be applied to task-evoked fMRI data. In the latter case, one or more spatially independent components, and their associated time course, will approximate the results of a standard modelbased group analysis (McKeown et al., 1998; Rombouts et al., 2009). In addition, when the time course of the task is kept identical across subjects, T-PICA also yields a subject-specific mode quantifying how much a certain spatio-temporal process is expressed by each subject (Beckmann and Smith, 2005; Rombouts et al., 2009), and can therefore be used to estimate the presence of different subgroups of participants contributing in different ways to different task-related spatio-temporal processes.

\section{Methods}

\subsection{Participants}

We acquired anatomical and functional magnetic resonance (MR) images on a sample of 45 healthy adult participants (age range: 20 to 47 years, median: 29.5 years) with no history of neurological disease, neuropsychiatric disorder or substance abuse. The sample was composed of 22 male and 23 female participants with 29 being right-handed and 16 left-handed. Self-reports of handedness were confirmed by the Annett test (Annett, 1970) in 35 participants. The study was performed in conformity with the declaration of Helsinki, and its protocol approved by the local Institutional Ethics Committee. All participants signed a written informed consent in compliance with the guidelines of the Institutional Review Board.

\subsection{MR image acquisition}

Functional magnetic resonance imaging (fMRI) was carried out during performance of a verb generation (VGEN) task as previously reported (Bizzi et al., 2008). A block design with nine 24-sec alternating periods and two conditions was used. In the experimental condition participants saw appearing on the screen the noun of an object of everyday use (e.g. "knife", "key", "scarf"). The list of nouns appears to contain in the vast majority those associated with a 'low selection' condition according to (Thompson-Schill et al., 1997); nouns with few associated responses or with a clear dominant response. In response to that, they had been instructed to covertly generate a verb describing the most appropriate action to be performed with the presented object. The covert modality was preferred to overt speech production to prevent the fMRI data from being contaminated by head and mouth motion artifacts, which would have been difficult to regress out, given their synchronization with the predictor of the task. The same list of words was presented in the same order to all the participants. In each experimental condition, eight stimuli were presented for two seconds each. Between each stimulus, a blank screen with a central black dot was presented for one second. During the rest condition patients were asked to fixate on a cross 
at the center of the screen. Stimuli presentation started and ended with one block of rest. Four such experimental blocks were acquired.

Brain images were acquired on a Siemens Avanto 1.5T MR scanner (Siemens, Erlangen, Germany). FMRI images were acquired using a T2*-weighted BOLD echo-planar imaging (EPI) gradient-echo sequence (TR/TE: $3000 / 52 \mathrm{msec} ; 256 \mathrm{~mm}^{2}$ field of view (FOV), $128 \times 128$ matrix and nominal resolution $2.0 \times 2.0 \times 4.0 \mathrm{~mm}^{3} ; 25$ sections with no intersection gap). A series of 76 volumes that included the first four initial dummy images was obtained in the transverse plane parallel to the anteroposterior commissural line. In addition, we also acquired a high-resolution anatomical image of each participant's brain using a sagittal volumetric T1-weighted magnetization-prepared rapid acquisition gradient-echo (MPRAGE) sequence (repetition time (TR)/echo time (TE)/inversion time (TI): 1640/2.28/552 msec; flip angle: $12^{\circ} ; 160$ sections with iso-volumetric resolution of 1.0 $\left.\mathrm{mm}^{3}\right)$.

\subsection{Analytical strategy}

Our aim was to assess whether the fMRI data acquired during the VGEN task would suggest the presence of different subgroups of participants using different patterns of brain regions recruited to perform the task. To this end, we used a model-free approach (Tensorial extension of Probabilistic Independent Component Analysis, hereafter T-PICA (Beckmann and Smith, 2005)) to (1) identify the presence of different spatio-temporal processes related to the execution of the task, and (2) quantify each subject's expression of these processes (see Fig. 1). This approach yields a functional network fingerprint (FNF) for each subject: a vector composed by concatenating the ICA-derived values quantifying the expression of each spatial process that is temporally associated with the task. We hypothesized that subgroups of participants would differ in their FNF. These fingerprints were then fed into a repeated clustering procedure, in order to define the subgroups. Finally, we performed a standard GLM analysis of group differences between the identified subgroups.

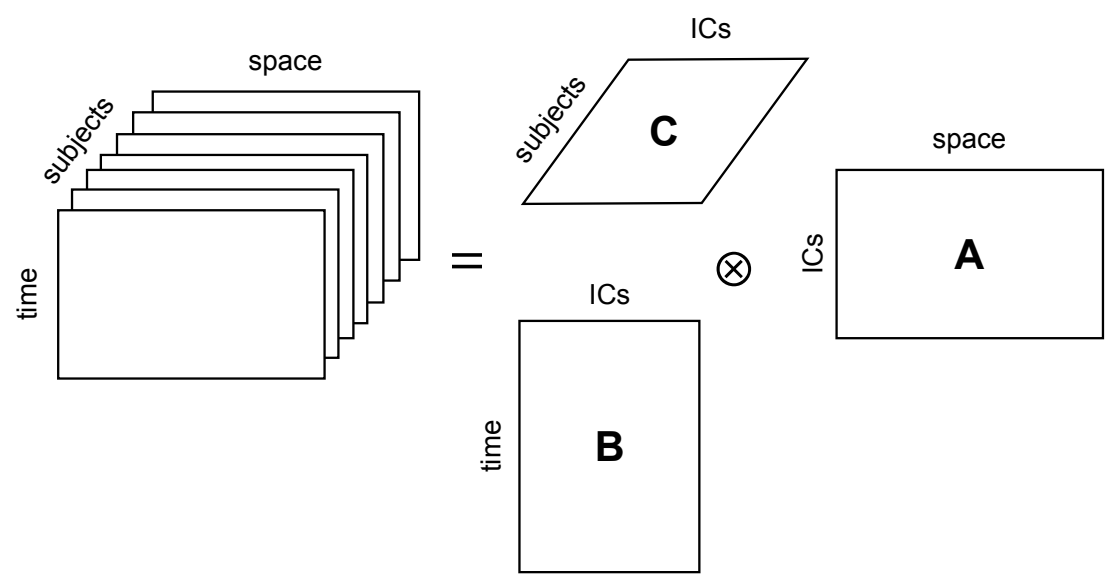

Fig. 1 - Graphical representation of the tensorial extension of ICA (T-PICA, (Beckmann and Smith, 2005)). The matrix of fMRI signal at each brain voxel, time-concatenated across subjects, is decomposed into three matrices describing (A) spatially independent components, (B) their associated characteristic time course, and (C) the relative amplitude of the spatio-temporal processes $(\mathbf{A} \times \mathbf{B})$ in each participant. The latter quantity was considered in the spatial components whose time course was associated with the execution of the task (see Fig. 2) and did not reflected nuisance variables such as motion. The values in the matrix $\mathbf{C}$ - one for each spatio- 
temporal process, for each subject - were concatenated in a functional network fingerprint (FNF), which was then fed into repeated k-means clustering to detect the presence of different subgroups of participants.

\subsection{MR Image Analysis}

\subsubsection{Preprocessing}

All image analyses were performed using FSL 5.0.5 (Smith et al., 2004; Woolrich et al., 2009). Structural (T1weighted) images were skull-stripped (and the results visually checked) after inhomogeneity correction. FMRI data were preprocessed with motion correction, slice-timing correction, spatial smoothing (Gaussian kernel, $F W H M=6 \mathrm{~mm}$ ) and highpass temporal filtering (100s). Each subject's fMRI image was then linearly registered to the MNI152 template via the high-resolution structural image, and resampled to a voxel dimension of $4 \times 4 \times 4 \mathrm{~mm}$. The resulting preprocessed data were subsequently used for T-PICA.

\subsubsection{Tensor Probabilistic Independent Components Analysis (T-PICA)}

T-PICA (Beckmann and Smith, 2005) was performed using FSL Melodic (Beckmann et al., 2005). The preprocessed $\mathrm{FMRI}$ data were whitened and projected into an n-dimensional subspace using Principal Component Analysis (PCA). The number of principal components for the current dataset was estimated to be 17 by using the Laplace approximation to the Bayesian evidence of the model order (Minka, 2000; Beckmann and Smith, 2004). Therefore T-PICA decomposed the data into 17 components, by optimizing for non-Gaussian spatial source distributions using a fixed-point iteration technique (Hyvarinen, 1999). Estimated component maps were divided by the standard deviation of the residual noise and thresholded at $P>0.5$ (Beckmann et al., 2003b) by fitting a mixture model to the histogram of intensity values (Beckmann and Smith, 2004).

T-PICA decomposes the fMRI data into spatial maps, associated time courses, and respective subject modes. Spatial maps (matrix A in Fig. 1) reflect the voxels that co-vary in time, and the principal component of the temporal dynamics is captured in their associated time course (matrix B in Fig. 1). The time course relative to a certain spatial map allows assessing the association of that pattern of fMRI signal with the execution of the task. Some of these spatio-temporal processes reflect haemodynamic fluctuations associated with task execution, others reflect some form of structured noise due to motion or other physiological or scanner-related artifacts (Salimi-Khorshidi et al., 2014; Pruim et al., 2015). Finally, the subject modes (matrix C in Fig. 1) represent a measure of how much a spatio-temporal process is expressed by each subject. Therefore the latter can be used to assess to what extent the extracted spatio-temporal processes are representative of the entire group, or are different between groups, or are expressed only in a single or a few subjects (Rombouts et al., 2009). In particular, by considering together all the spatio-temporal processes which have a high correlation with the task, we obtain a multidimensional estimate of how much each participant expressed different spatio-temporal patterns: we denote this a 'functional network fingerprint' (FNF). Should subgroups be present, the FNF of each subgroup's participants will be more similar to each other than to those of other subgroups. One way to assess this is by means of a clustering analysis, as detailed below.

\subsubsection{Definition of subgroups and estimation of differences among subgroups}

Out of the 17 spatio-temporal processes extracted by T-PICA, we considered nine processes, which presented a time course highly correlated with task execution (see Fig. 2) and whose activity was mostly located in the grey matter. We did not further consider (1) processes whose spatial map reflected motion or other physiological artifacts, which are commonly extracted by means of ICA decomposition (Salimi-Khorshidi et al., 2014; Pruim et al., 2015); (2) processes whose subject modes would display high values in only one subject. The subject modes associated with the nine processes were concatenated as one vector representing the FNF for each subject. 
Finally, the matrix of all subjects' FNF was fed into the k-means algorithm implemented in MATLAB (The Mathworks Inc. Natick, MA) to extract clusters of participants featuring internally homogeneous and reciprocally different FNFs. To prevent the possibility that the retrieved clustering solution could be driven by the initial random placement of the cluster centers, the clustering procedure was repeated 1000 times. The results of this repeated clustering was used to determine the most appropriate number of cluster to ask the algorithm: for each choice of cluster number in the range from 2 to 10 , the stability of the clustering solution was estimated from the rank of the matrix concatenating the cluster labels for each repetition. We anticipate that the most stable solution, which returned the same cluster assignment over 1000 repetitions, was the one yielded by asking for three clusters. We therefore concluded that the FNF supported the definition of three subgroups of participants in the initial dataset. To characterize the three subgroups of participants in terms of regional differences in the taskrelated fMRI signal, as in previous works (Noppeney et al., 2006; Kherif et al., 2009) we performed a modelbased voxel wise group comparison. This analysis was carried out in the framework of the general linear model (GLM) using FSL FLAME (Beckmann et al., 2003a). In this analysis age, sex and handedness were modeled as covariates-of-no interest.

In most of the neurotypical population, language production tasks such as VGEN have been demonstrated to yield highly left-lateralized brain responses that are almost independent from handedness (Mazoyer et al., 2014). Despite this, handedness represented one of the main sources of variability in our sample, and following the advice of the reviewers we examined whether differences in brain activity during VGEN between ICA-derived subgroups could have been driven by handedness. We thus estimated differences between right- and left-handed participants in the whole sample during the execution of the task, and performed a voxel-wise regression of the parameter estimates associated with the task against the Annett scores for the 35 participants for whom this information was available. We also performed a regression analysis of the task-related parameter estimates against participants' age, and we estimated group-level differences between males and females, with the same aim of assessing to what extent differences between the detected subgroups could be explained in terms of the available demographic sources of inter-subjective variability. For all the described analyses, we will report effects that are significant at $\mathrm{P}<0.05$ and FWE-corrected at the cluster level using Gaussian Random Field theory (Worsley et al., 1996; Woolrich et al., 2004).

\section{Results}

\subsection{Spatio-temporal processes extracted by T-PICA analysis}

Tensor-based probabilistic ICA decomposed the fMRI dataset in 17 spatio-temporal patterns, and their associated subject modes. Out of them, the first nine components satisfied our criteria for inclusion in the following analyses: (1) highly correlated with the task (See Fig. 2); (2) mostly distributed in the gray matter; (3) substantially expressed by a consistent number of participants; (4) clearly distinguishable from typical components reflecting motion, cardiac pulsation and other sources of physiological artifacts (See Fig. S1). The spatial maps associated with the first seven components (IC1-IC7) all included the left inferior frontal gyrus (IFG) and the left premotor cortex (PMC) including the medial preSMA region. Besides IFG and PMC, these maps encompassed different combinations of other brain regions commonly found in studies examining (1) verb generation (posterior parietal lobule, bilaterally); (2) reading single words (extrastriate visual cortices and occipitotemporal regions, bilaterally). IC8 encompassed a right-lateralized temporo-parieto-frontal network involved in attention and working memory, whose activity was increased during the cross fixation, while IC9 was only expressed in the temporo-occipital cortex bilaterally, immediately posterior to the so-called visual word form area (Dehaene and Cohen, 2011). Several of these components also described fMRI signal negatively correlated with the task, such as for regions in the default mode network (especially expressed in IC2) and in the bilateral primary sensory and motor cortices (IC6). The subject modes associated to the spatio-temporal processes present in the 9 selected components were considered together in a subject-specific functional network fingerprint (FNF), quantifying how much a subject would express each of the nine task-related components. 

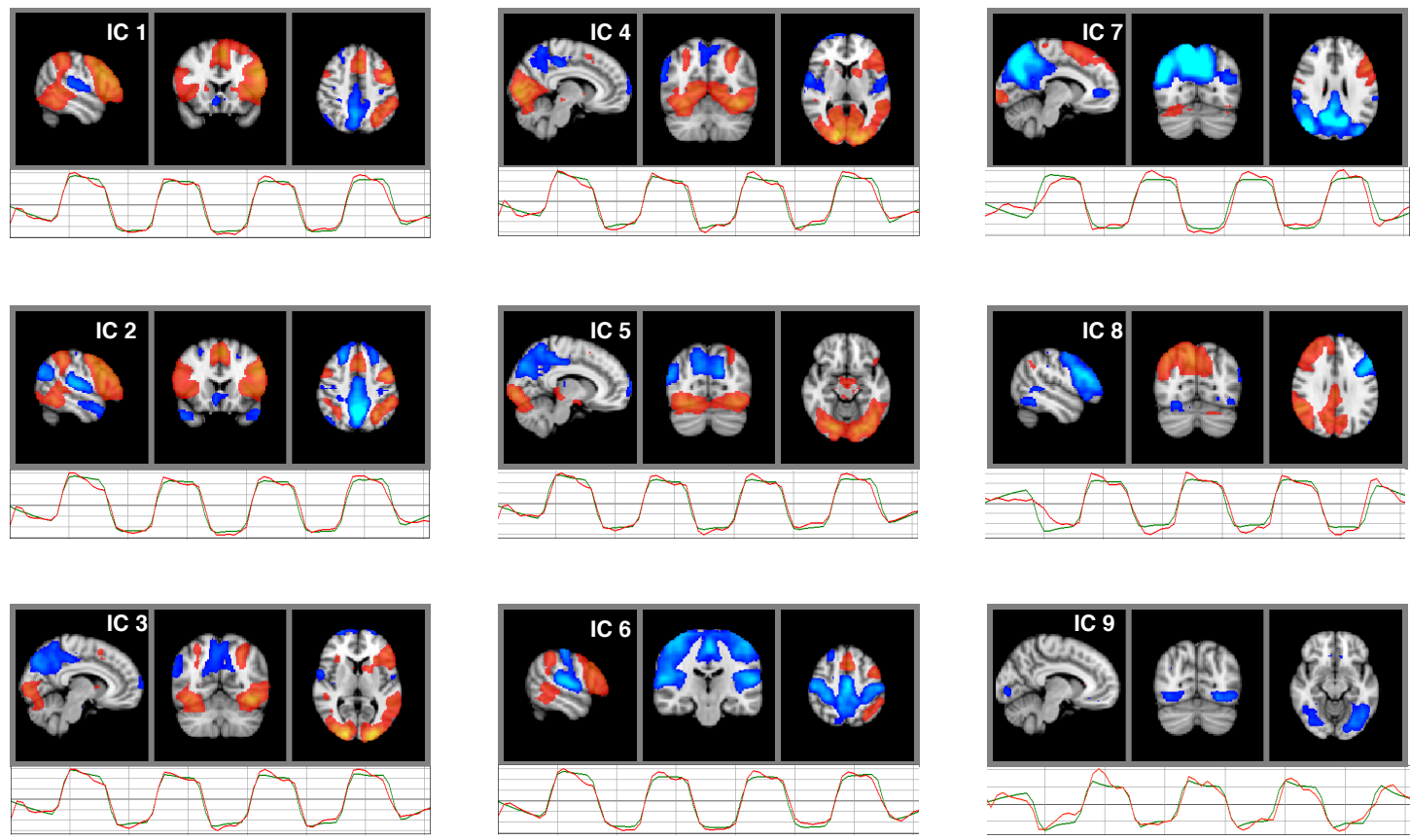

Fig. 2 - Spatio-temporal processes associated with the VGEN task, displayed in radiological orientation. The components are ordered according to the decreasing amount of total variance explained in the fMRI data. The displayed values of the component maps have been thresholded by fitting a mixture model to the histogram of intensity values (Beckmann and Smith, 2004). Below each spatial map, the associated time course, averaged across participants, is shown in red. In green, we show the convolved boxcar design describing the time course of the experimental paradigm. The same boxcar design was used for every participant, as required by T-PICA for comparing the subject-specific modes across participants. For IC1-IC7, red-yellow overlays are associated with a positive correlation of the pattern of activity with the VGEN task, while blue-azure overlays are associated with a negative correlation with VGEN. For IC8-IC9, the situation is reversed. Flipping the sign of the component maps does not change the meaning of the results, and is merely due to the fact that the T-PICA decomposition is invariant under inversion of two of the three modes it extracts (see (Rombouts et al., 2009)). The excluded components are presented in Fig. S2.

\subsection{Definition of the subgroups and estimation of between-subgroups differences}

The matrix of all participants' FNFs was repeatedly fed into the k-means clustering algorithm to determine the presence and distribution of subgroups of participants. Across the range from 2 to 10 clusters, only for the 3cluster solution was the matrix rank exactly three, indicating that the assignment of different participant's FNF to different clusters/subgroups had been consistent across all the 1000 repetitions. This solution partitioned the sample of participants in three subgroups sg1, sg2 and sg3 with numerosity $N_{s g 1}=6, N_{s g 2}=13$, and $N_{s g 3}=23$ respectively (see Table 1). The three subgroups did not significantly differ in the proportion of right- and lefthanded participants $\left(X^{2}(2)=4.70, p=0.1\right)$, sex $\left(X^{2}(2)=0.88, p=0.64\right)$ or age $\left(F_{(2,42)}=0.33, p=0.72\right)$. However, the GLM analysis of task-related haemodynamic response in the brain revealed a distributed set of significant differences between the three subgroups (See Fig. 3). Sg2 and Sg3 shared some differences with respect to Sg1: the participants in $\mathrm{Sg} 1$ showed higher activity in bilateral extrastriate visual regions and in the left inferior frontal gyrus. Sg2 and Sg3 showed higher activity in the right inferior posterior parietal lobe as well as in the medial and 
lateral BA9. Finally, Sg2 and Sg3 showed reciprocal differences restricted to the precuneus bilaterally and the left occipital pole (see Fig 3).
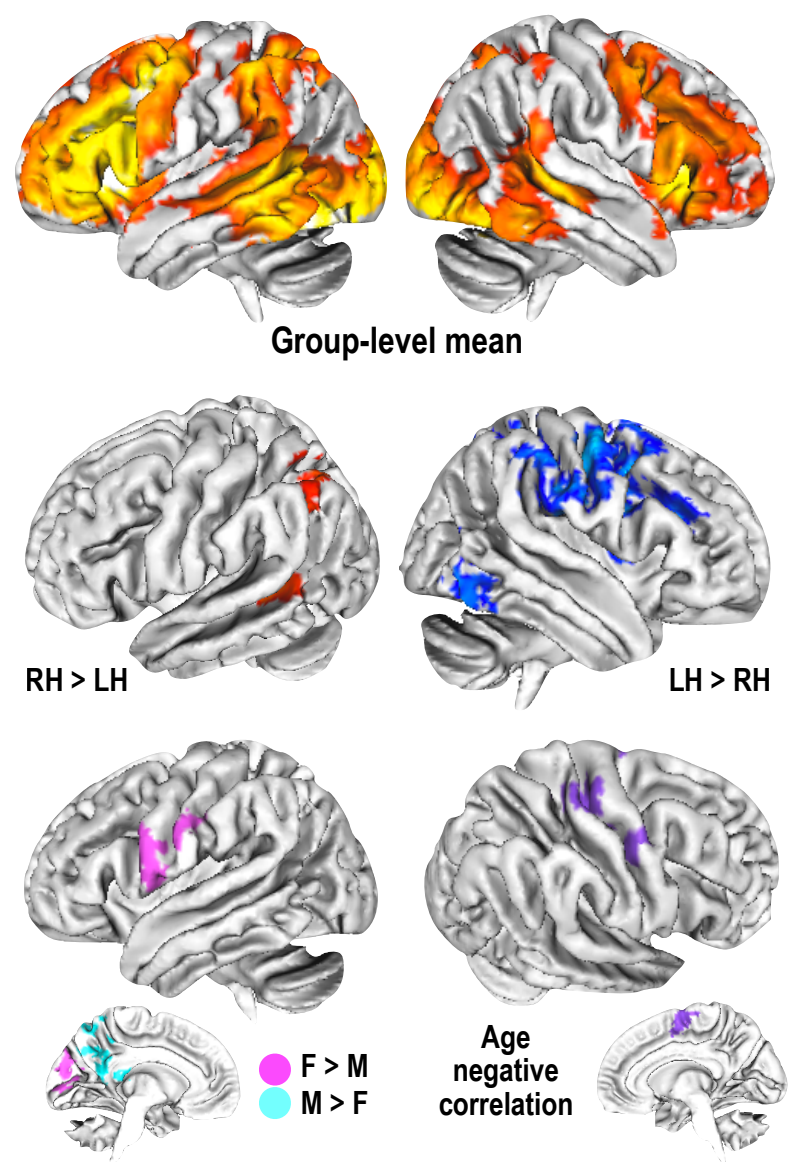
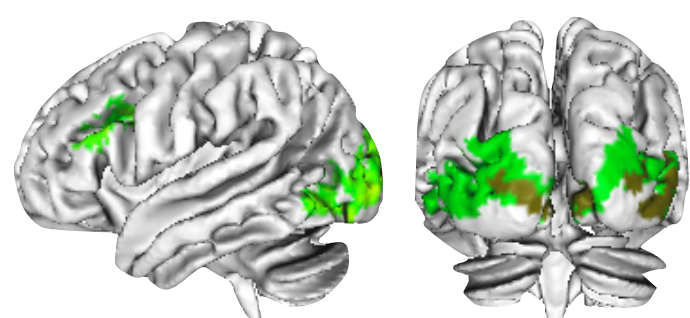

$\mathrm{Sg} 1>\mathrm{Sg} 2$

$\mathrm{Sg} 1>\mathrm{Sg} 3$

overlap
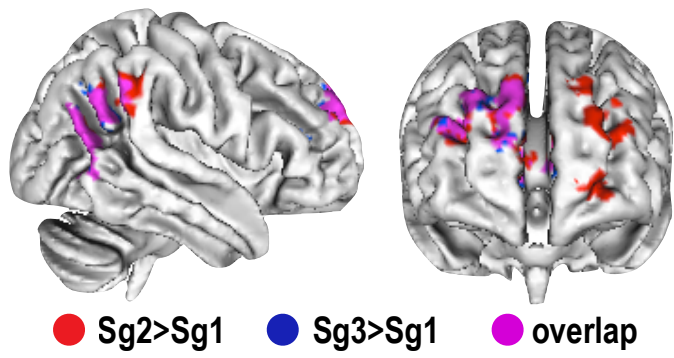

$\mathrm{Sg} 2>\mathrm{Sg} 1$

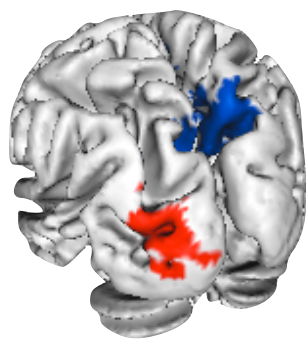

$\mathrm{Sg} 2>\mathrm{Sg} 3$

$\mathrm{Sg} 3>\mathrm{Sg} 2$

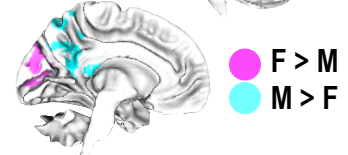

Fig. 3 - Left panel: The top row shows common activation pattern yielded by a group-level analysis on the whole sample of 45 participants, by controlling for age, sex and handedness. The middle and bottom row show which regions were associated with differences in terms of handedness $(\mathrm{LH}=$ left-handed; $\mathrm{RH}=$ right-handed participants), sex ( $F=$ Females; $M=$ Males) in the entire sample. No brain region was positively associated with Age. Right panel: Brain regions where a significant difference in the parameter estimates of task-related activity was found across the three subgroups ( $\mathrm{Sg} 1 \mathrm{~N}=6$; $\mathrm{Sg} 2 \mathrm{~N}=23$; $\mathrm{Sg} 3 \mathrm{~N}=16$ ) identified by performing k-means clustering on the subject-specific modes of the ICA components. This analysis was performed using additional covariates to control for age, sex and handedness. Note how the patter on between-subgroup differences is completely dissociated from those yielded by contrasting right- with left-handed participants, showing that the former cannot be explained by the different composition of the subgroups in terms of handedness. Analyses of the demographic variables showed that the three subgroups did not differ in terms of mean age and proportion of males/females or right-/left-handed participants.

\subsection{Differences associated with handedness, age, and sex}

Significant differences in task-related brain activity between right- $(\mathrm{RH})$ and left-handed $(\mathrm{LH})$ participants were detected in different locations with respect to those highlighted by the contrasts between the three subgroups. In right-handed participants, VGEN parameter estimates were higher than left-handed participants in regions of the left posterior parietal cortex adjacent to the intraparietal sulcus, as well as in the left posterior STS (RH>LH in Fig 3). For the $\mathrm{LH}>\mathrm{RH}$ contrast, a significant effect was found in several left-hemispheric parietal and frontal regions, as well as in the posterior inferior temporal gyrus. Regression of the task-related parameter estimates against the 
Annett handedness scores yielded a significant effect in the regions where the effect of handedness was more prominent when right- and left-handed participants were compared with each other (see Fig. 3 and Fig. S2). Females displayed stronger activity than males in the left ventral premotor and somatosensory region, as well as in the medial occipital lobe, while the reverse contrast yielded higher activity in the left precuneus. Finally, age was negatively correlated with task-related activity in the right primary motor and somatosensory cortex.

\section{Discussion}

\subsection{Main findings}

In the present study we applied Tensor-based Probabilistic Independent Component Analysis (T-PICA, (Beckmann and Smith, 2005)) to the fMRI data of 45 healthy adult participants acquired while they were performing the verb generation task (VGEN). We decomposed the signal associated with the execution of the VGEN task as different spatio-temporal processes, and quantified how much each subject expressed these processes. Our aim was to assess the presence of subgroups of participants characterized by different spatial patterns of brain activity between each other, and with respect to the group-level results. To this end, we performed a cluster analysis on vectors quantifying differences in the subject-specific contribution to different task-related spatio-temporal processes.

Three main findings emerged from our analyses. (1) T-PICA decomposed the task-related fMRI signal into nine spatio-temporal processes, some of which (IC6, IC8) were relatively unexpected, thus providing a richer characterization of the brain activity during VGEN with respect to what has been reported in previous modelbased fMRI studies employing this task. (2) Clustering analyses of the subject-specific expression of these spatiotemporal modes robustly revealed the presence of three distinct subgroups of participants, characterized by different levels of haemodynamic response to the task in prefrontal, posterior parietal and extrastriate occipital regions. (3) Differences in brain activity between subgroups could not be explained in terms of age, sex or handedness.

\subsection{Comparison of T-PICA spatial components with previous model-based fMRI findings obtained using the VGEN paradigm.}

The vast majority of task-related spatial components extracted by T-PICA were located as expected, in a frontoparieto-temporal network described in previous fMRI works using the VGEN task (Grezes and Decety, 2001; Holland et al., 2001; Karunanayaka et al., 2010; Horowitz-Kraus et al., 2014). Within this network, activity in the left IFG is related to the cognitive load imposed by the task (Thompson-Schill et al., 1997; Persson et al., 2004; Berlingeri et al., 2008). The observed activity in posterior temporal regions bilaterally, involved in the retrieval of semantic memories, appears to be modulated by the degree of repetition of semantic information (ThompsonSchill et al., 1999). The rostral part of the bilateral medial premotor regions (preSMA), also commonly found during the VGEN task, is known to contribute in higher-order associative processing of sensory stimuli for the selection of motor plans (Picard and Strick, 2001). In addition, studies in primary progressive aphasia (GornoTempini et al., 2004) show that the entity of damages to the frontal aslant tract (Catani et al., 2012), connecting preSMA and Broca's region, is associated with deficits in verbal fluency (Catani et al., 2013). Finally, task-related changes in the haemodynamic response were also found in several regions of the posterior parietal cortex (PPC) bilaterally, and more extensively in the left hemisphere. In the context of VGEN, PPC is likely to play a multimodal role. Lesion studies have shown the importance of PPC in storing and accessing motor representations (Leiguarda and Marsden, 2000), however its involvement is also likely linked to the necessity of holding the noun of the object in the working memory during verb generation (Karunanayaka et al., 2011).

Besides these regions, T-PICA extracted other components reflecting task-related activity, which are not commonly reported in VGEN studies. IC8 shows higher activity during cross fixation in a right-lateralized network, commonly found using T-PICA on resting-state fMRI data (Damoiseaux et al., 2006; Smith et al., 2009) and 
involved in visuo-spatial attentional tasks (Thiebaut de Schotten et al., 2011). IC6 is more interesting with respect to the specificity of the verb generation task, because it shows that activity in primary sensory and motor regions in both hemispheres was decreased during the task with respect to the periods of cross fixation. Given the high correlation of these 'deactivations' with the VGEN task, it is unlikely that they are associated with brain activity related to the usual instruction given to the participants to remain still during the experiment. Instead, they are more likely to reflect a selective inhibition of the final motor pathway in fronto-parietal networks involved in execution, simulation, and observation, and possibly also verbalization of motor activities (Grezes and Decety, 2001; Berlingeri et al., 2008)

\subsection{On the nature of the differences between subgroups}

Our analyses highlight the presence of three subgroups $(\mathrm{Sg} 1, \mathrm{Sg} 2, \mathrm{Sg} 3)$ of participants characterized by either different levels of activity within the group-level pattern, or by activations located in different brain regions above the group-level map, after controlling for age, sex and handedness. To simplify the exposition of betweensubgroup differences, we will denote increased or decreased task-related parameter estimates as increased or decreased 'activation'. Sg1 showed increased activation in bilateral extrastriate occipital regions with respect to both Sg2 and Sg3 (Fig. 3, right panel). This effect extended to cover almost the entire dorsal extrastriate occipital cortex bilaterally for the contrast Sg1>Sg2. Additionally, Sg1 showed higher activity than Sg2 in the left inferior frontal sulcus, extending onto the left pars triangularis. The reverse contrast showed that both $\mathrm{Sg} 2$ and $\mathrm{Sg} 3$ featured higher activation in the right angular gyrus and in the right rostral superior frontal gyrus (putative BA9). In addition to this pattern, Sg2 showed higher activity than Sg1 in the right posterior supramarginal gyrus and in the left rostral superior frontal sulcus, extending to the adjacent frontal gyri and to the frontal pole. Finally, Sg2 featured higher activation than $\mathrm{Sg} 3$ in the left dorsal occipital extrastriate region, while the reverse contrast (Sg3>Sg2) showed higher bilateral activation in the precuneus. Importantly, all the regions where a significant difference in activation was elicited by the contrasts $\mathrm{Sg} 2>\mathrm{Sg} 1, \mathrm{Sg} 3>\mathrm{Sg} 1$ and $\mathrm{Sg} 3>\mathrm{Sg} 2$ had not been displayed in the group-level analysis on the entire sample.

The variability associated with functional activations between subjects is classically attributed to noise (Noppeney et al., 2004). However, distinct patterns of task-related brain activity feature a remarkable longitudinal stability across repeated fMRI sessions separated by several months (Miller and Van Horn, 2007; Miller et al., 2012). Also, it has recently been shown that inter-subject variability in task-related brain activity is accurately predicted by resting-state fMRI data (Tavor et al., 2016). Such evidence suggests that variability in functional activations could be grounded in real differences across subjects (Price and Friston, 2002). This is important as standard group-level analyses might conceal these differences and yield a significant association with the task only in those regions shared by the vast majority of participants in the considered sample. The latter situation is apparently depicted in our results: some regions where a significant difference in the task-related parameter estimates was found between subgroups were also present in the group-level results, such as in the left IFG and in the extrastriate occipital regions; however right posterior parietal and bilateral dorsal prefrontal regions, where activity was increased in $\mathrm{Sg} 2$ and $\mathrm{Sg} 3$ with respect to $\mathrm{Sg} 1$, were not reported as significant by the analysis on the entire sample (see Fig. 3). The nature of this variability is difficult to establish given the data at hand. While subgroups of participants did not significantly differ in terms of age, sex and handedness, we assessed whether the effect of these variables on the entire sample could have driven some of the differences between the subgroups. We found that all three demographic variables had an effect on the task-related haemodynamic response, however such effect was present in different brain regions with respect to those elicited by contrasting different subgroups (see the comparison between right and left panel in Fig. 3). An analogous situation, where subgroup differences could not be entirely attributed to the recorded phenotypical variables, was reported in a previous study investigating brain activity during a reading aloud task (Kherif et al., 2009), where three out of four subgroups showing different pattern of brain activity did not differ in terms of the recorded demographic variables. Therefore, we cannot exclude that the detected subgroup differ along an unrecorded demographic dimension. In addition, it is difficult to provide a behavioural interpretation of the differences between the subgroups, since the 
presented data were not originally acquired for the purposes of the present analyses, but rather to validate the VGEN task against intracortical mapping in a clinical setting for the purposes of presurgical mapping of eloquent regions (Bizzi et al., 2008). Therefore we did not perform a debriefing of the participants after the fMRI examination aimed at assessing the cognitive strategy used to perform the task.

In conclusion, the detected differences in the pattern of activity between subgroups could be due to a number of reasons, including unrecorded demographic variables, network degeneracy (Price and Friston, 2002), anatomical differences or the use of different combination of strategies. Given the limited assessment of intersubject differences along these dimensions in our participants, the available data do not allow us to support one or the other of these possibilities. However, our results highlight the presence of a source of inter-subject variability, which led to detect significant differences in the pattern on brain activity that would have otherwise gone unnoticed at the group level.

\subsection{Comparison of T-PICA with previous methods proposed to detect the presence of spatial heterogeneity in} patterns of activation between subgroups of participants

As already mentioned in the Introduction, a few previous papers dealt with the problem of assessing the presence of subgroups of participants showing patterns of task-related activation departing from the group-level mean (Noppeney et al., 2006; Seghier et al., 2008; Kherif et al., 2009). The main idea in previous attempts to demarcate the differences between individuals was to use a singular value decomposition (SVD) of the residual variance from a group-level parameter estimate of the task-related contrast of interest, that is on the subjectspecific deviation from the group mean (Noppeney et al., 2006); a mass univariate voxelwise approach was employed to obtain one residual variance map per subject. These maps were concatenated across subjects in a matrix of dimension M-by- $\mathrm{N}$, where $\mathrm{M}$ is the number of subjects and $\mathrm{N}$ the number of voxels in brain. The SVD produces a decomposition in terms of eigenimages and eigenvariates which represent the activation pattern in the brain, and the extent to which each subject reflects that pattern, respectively. Similarly in (Kherif et al., 2009), the authors perform a principal component analysis (PCA) on the same residual maps in order to represent the data in a lower dimensionality, following which they invoke a generative model to ascertain the pattern of activities that emerge out of $k$ distinct components. A different approach to study inter-subject variability was implemented in (Seghier et al., 2008). There, it was hypothesized that subjects would differ, beyond a group-level pattern, in the relative activation of brain networks centered on anterior or posterior left occipito-temporal regions as defined in previous studies. To this aim, the authors performed a group-level multiple regression on the subject-level parameter estimates of a reading aloud task, using the mean parameter estimates in either regions of interest as predictors. Importantly, these authors found that the inter-subject differences in the activity of either networks correlated with the response time to read irregular words and pseudowords. The rationale of this study is different from those of (Noppeney et al., 2006; Kherif et al., 2009) and from ours, since it took the lead from an hypothesis grounded in two specific brain regions, rather than estimating the presence of subgroups on the basis of the whole maps of parameter estimates. In other words, it is possible that our method and those of (Kherif et al., 2009) and (Noppeney et al., 2006) could have retrieved similar or different partitions of the groups of individuals, by looking at the covariation of the (residuals of) parameter estimates across the whole brain. Therefore in the following we will focus our methodological comparison to considering the algorithms implemented in (Kherif et al., 2009) and (Noppeney et al., 2006). We propose that the method we implemented in the present study, based on T-PICA, bears some advantages with respect to the aforementioned residual-based methods. These advantages can be summarized in three points:

(1) While in principle there can be consistent between-group differences in the residuals of the taskrelated parameter estimates, they do not necessarily reflect differences in the neuronal signal related to taskexecution. Instead they could be caused, at least in part, by motion, physiological artifacts, scanner drifts. This is particularly evident for the case of motion: in a standard GLM analysis (such as those performed in (Noppeney et al., 2006) and (Kherif et al., 2009)) task-related motion will inflate the parameter estimates associated with the task. The signal associated with motion can be mixed with neuronal signal across the whole brain, however, it is 
particularly pronounced in specific locations at the outer border of the brain (Salimi-Khorshidi et al., 2014). Since our T-PICA is optimizing components extraction for spatial independence, it is capable of isolating such motionrelated components even in the presence of a temporal correlation with the task. By removing the time course associated to these spatial component, it is possible to unmix the motion-related signal from that related to the task in every brain voxel before estimating the task-related parameter estimates in the cortex and in deep nuclei (Pruim et al., 2015). In the case of motion-related noise orthogonal to the task-related signal, the GLM regression coefficient associated with the task will not be affected, however the residual variance with respect to the task predictor will be inflated (Beckmann, 2012). In addition, recent studies carried out in a large cohort of subject and across different task-evoked fMRI data showed that estimating motion-related signal from the data using ICA, with respect to explicly modeling it using motion-parameter estimates, increases the sensitivity to activations and deactivations, and reduces the estimated activity around brain edges (Pruim et al., 2015). Therefore ICA allows to remove the variability in the residuals of the model which can result in both artefactually increased or decreased task-related parameter estimates.

(2) The standard GLM analysis performed in the previous studies rests on the assumption that the BOLD response is the same for all participants, while it has been shown that the haemodynamic response can vary across neurotypical individuals (D'Esposito et al., 2003; Handwerker et al., 2004; Schippers et al., 2011), as well as in individuals affected by neurodegenerative diseases (Rombouts et al., 2005). In these cases, the interpretation of the residual variance from group-level parameter estimates remains ambiguous, since it could be due either to the deviation from the standard haemodynamic response function used for all subjects, or to the level of task-related activity. With respect to this, it has been shown that T-PICA is capable of detecting such differences in the haemodynamic response (Rombouts et al., 2009), and therefore to yield a more precise estimation of each subject's contribution to the group-level pattern of task-related activity.

(3) Finally, the crucial difference between our analysis and that carried out in the aforementioned studies is that in all of them the temporal activities of the subjects was collapsed over time in order to obtain the regression coefficients. In other words, the activity over time was summarized into the regression coefficients for further analysis, deeming them sensitive to perturbations in temporal dynamics only to the first order. For example, if a subject co-varied with the task only in the first half and another subject co-varied with the task in the second half, both of them would have similar coefficients, assuming that they co-varied in the same manner in the first and second halves respectively. This can lead to lose valuable information on the temporal aspect of the covariation between these two subjects. Herein lies the difference between T-PICA and other methods. Within TPICA, the template used to extract subject-wise contribution is composed of matrix formed by the outer product of $\mathbf{B}$ and $\mathbf{A}$ (see figure 1). This outer product provides a much richer representation by combining spatio-temporal information into the template thus making it more sensitive to higher order temporal variations whilst performing the task. This provides additional information to disentangle an individual's contribution to the group-level pattern of activity.

\subsection{Applications of model-free methods for detection of subgroups and other sources of inter-subjective variability.}

While in the present work we analyzed fMRI data acquired in a sample of healthy adult controls, T-PICA as well as other model-free methods for detection of subgroups of participants are likely to be particularly useful in analyzing samples of patients diagnosed with a neuropsychiatric condition, with the aim of detecting different subtypes (Fair et al., 2012). In this respect, one recent work (Gates et al., 2014) used graph theoretical measures (community detection, (Newman, 2006)) to detect the presence of subgroups of children diagnosed with ADHD based on resting-state fMRI data. In this study, the presence of subgroups was hypothesized to be related to differences in the interaction between the nodes of the same network, rather than to a heterogeneous expression of different brain networks. Different subgroups of ADHD participants featured either increased or decreased functional connectivity between posterior parietal and dorsolateral prefrontal regions, which would have likely been undetected by pooling together all the participants. Importantly, while the ratio between ADHD and control 
participants was greater than by chance, all subgroups included both ADHD and control participants. Applying the presented method based on T-PICA to such datasets would allow detecting whether different subtypes could be characterized also in terms of the expression of different functional networks, as well as in their interaction (Jafri et al., 2008; Cerliani et al., 2015)

Another remarkable application of model-free methods such as ICA is represented by the possibility of detecting differences between categories of participants with different risks of developing a degenerative neurological syndrome, and to do so in an early and even presymptomatic stage. For instance, an ICA-based examination of the spatio-temporal properties of the Default Mode Network (Fox et al., 2005) led to detect differences in the intrinsic functional architecture of the brain in young asymptomatic adults who were the carrier of the APOE- $\varepsilon 4$, which has been shown to be associated with a high risk of developing Alzheimer's disease (Filippini et al., 2009). Finally, in studies involving aging and degenerative diseases, the added value of modelfree techniques is given by the capability to account for deviations of the fMRI signal with respect to the expected haemodynamic response function used in model-based approaches to estimate task-dependent regional activity (D'Esposito et al., 2003; Rombouts et al., 2005). In this context, the subject-specific modes of T-PICA were used to show that demented participants were characterized by a decrease in the activation and deactivation of taskrelated functional networks. In addition, such measures were able to detect group differences in twice as many networks than by using model-based approaches (Rombouts et al., 2009).

\subsection{Limitations}

Using T-PICA and other model-free methods to detect the presence of subgroups and other sources of intersubject variability comes at the cost of a number of limitations that also apply to the present study.

While the use of ICA to denoise the data (Beckmann, 2012) increases the confidence that subgroups detected using this strategy are unlikely due to noise, the interpretation of what might have led to the such subgroups can be hard. The observed source of variability might relate to (1) demographic and/or behavioural traits that are not specific for the task under investigation (Smith et al., 2015), (2) reflect the presence of degenerate neural system sub-serving the same function (Price and Friston, 2002), or (3) suggest the possibility that different subgroups implemented different cognitive strategies to perform the task (Noppeney et al., 2004; Noppeney et al., 2006; Jobard et al., 2011; Miller et al., 2012). Each one of these interpretations, and the decision to support one or the other alternative, requires a process of reverse inference (Poldrack, 2006) that should be used with caution, and be supported by an accurate behavioural assessment of participants. In our case, we only had access to basic demographic information about our participants and therefore we could not support a strong behavioural interpretation of the differences we detected.

Related to this, it is currently not possible to assess whether the presence of different patterns of activations reflects the implementation of different cognitive strategies in our verb generation task. On the one hand many variables can influence the patterns of activity elicited by verb generation tasks, such as the number of possible responses or the strength of association between the cued noun and verb associated to the appropriate action (Thompson-Schill et al., 1997; Martin and Cheng, 2006; Crescentini et al., 2010). On the other hand, most of the brain regions that are highlighted by our contrast between subgroups are involved in panoply of different tasks, such as the IFG and the inferior posterior parietal lobe. Therefore it is hard to estimate the specificity of their functional role during verb generation. For instance previous studies suggest that during different tasks requiring retrieval from the semantic knowledge, the differential activity of the IFG is linked to an increase in task demands, due to competing alternatives, rather than being specific to verb processing (Thompson-Schill et al., 1997; Berlingeri et al., 2008). On the basis of these considerations, and due to the lack of behavioural assessment in our participants, we refrained from commenting on the different patterns of activity in terms of different cognitive strategies, and we leave this issue for future studies.

On the methodological side, using the subject-specific modes of the spatio-temporal processes individuated by T-PICA in the way we described is only possible when analyzing task-evoked fMRI data where each subject 
has an identical study design for event types and timings (Rombouts et al., 2009). The reason for this is that the subject-specific modes yielded by T-PICA quantify the extent to which each individual expresses a certain spatiotemporal process, only if we can assume the invariance of these spatio-temporal processes across subjects. Therefore the use of T-PICA that we have described in the present work cannot be readily extended to the analyses of resting-state fMRI datasets such as the ABIDE (Di Martino et al., 2013). Further methodological investigation is granted to determine the meaningfulness of the subject-specific modes yielded by T-PICA for resting state data.

\subsection{Conclusions}

In conclusion, we presented an original data-driven approach to detect unexpected subgroups of participants in task-based fMRI data. With respect to previous works on this issue, the use of Tensor Probabilistic ICA allows us to discriminate which of the extracted patterns of activity relate to the task, and to isolate other sources of variability in the fMRI signal which are not temporally related to the task or are due to artifactual effects. Characterizing the participants based on their differential contribution to different task-related functional networks allowed us to discover three subgroups of participants featuring different parameter estimates of brain activity associated to the task in regions shared with the group-level pattern, as well as in other regions not found when all the participants were pooled together. This spatial heterogeneity reflected an unexpected source of intersubject variability that could not be explained by age, sex or handedness. We propose that data-driven approaches such as the one we presented can be useful to probe the hypothesized homogeneity of a sample of participants before conducting analyses of between-group differences along the expected dimensions of variability, for instance among clinical and healthy participants. If not considered, such unexpected sources of variability can undermine the ability to detect expected effects at the group level, as well as the interpretation of the obtained results. We believe that in the near future analytical approaches based on model-free methods like ICA will play a progressively more important role in the characterization of different subtypes of neuropsychiatric syndromes, as well as in monitoring the incipience of compensatory strategies in early stages of degenerative neurological syndromes.

\section{Acknowledgements}

This work was funded by grants ANR-13-JSV4-0001-01 and ANR-10-IAIHU-06 from the Agence Nationale de la Recherche. Leonardo Cerliani wishes to acknowledge Koen Haak, Daniel Margulies and Michel Thiebaut de Schotten for useful discussions during the preparation of this manuscript, as well as the Editor and the Reviewers for their insightful comments, suggestions and constructive critiques to the first version of the manuscript. Leonardo Cerliani wishes to dedicate this paper to the memory of his friend and research colleague Filippo Migliorati.

\section{DISCLOSURES}

None.

\section{References}

Annett, M (1970) A classification of hand preference by association analysis. British Journal of Psychology, 61:303-321.

Beckmann, CF (2012) Modelling with independent components. Neuroimage,

Beckmann, CF, DeLuca, M, Devlin, JT, Smith, SM (2005) Investigations into resting-state connectivity using independent component analysis. Philos Trans R Soc Lond B Biol Sci, 360:1001-1013. 
Beckmann, CF, Jenkinson, M, Smith, SM (2003a) General multilevel linear modeling for group analysis in FMRI. Neuroimage, 20:1052-1063.

Beckmann, CF, Smith, SM (2004) Probabilistic independent component analysis for functional magnetic resonance imaging. IEEE Trans Med Imaging, 23:137-152.

Beckmann, CF, Smith, SM (2005) Tensorial extensions of independent component analysis for multisubject FMRI analysis. Neuroimage, 25:294-311.

Beckmann, CF, Woolrich, MW, Smith, SM (2003b) Gaussian/Gamma mixture modelling of ICA/GLM spatial maps. Ninth Int Conf Funct Mapp Human Brain, 19(2):S985.

Berlingeri, M, Crepaldi, D, Roberti, R, Scialfa, G, Luzzatti, C, Paulesu, E (2008) Nouns and verbs in the brain: grammatical class and task specific effects as revealed by fMRI. Cogn Neuropsychol, 25:528-558.

Bizzi, A, Blasi, V, Falini, A, Ferroli, P, Cadioli, M, Danesi, U, Aquino, D, Marras, C, Caldiroli, D, Broggi, G (2008) Presurgical functional MR imaging of language and motor functions: validation with intraoperative electrocortical mapping. Radiology, 248:579-589.

Catani, M, Dell'acqua, F, Vergani, F, Malik, F, Hodge, H, Roy, P, Valabregue, R, Thiebaut de Schotten, M (2012) Short frontal lobe connections of the human brain. Cortex, 48:273-291.

Catani, M, Mesulam, MM, Jakobsen, E, Malik, F, Martersteck, A, Wieneke, C, Thompson, CK, Thiebaut de Schotten, M, Dell'Acqua, F, Weintraub, S, Rogalski, E (2013) A novel frontal pathway underlies verbal fluency in primary progressive aphasia. Brain, 136:2619-2628.

Cerliani, L, Mennes, M, Thomas, RM, Di Martino, A, Thioux, M, Keysers, C (2015) Increased Functional Connectivity Between Subcortical and Cortical Resting-State Networks in Autism Spectrum Disorder. JAMA Psychiatry, 72:767-777.

Crescentini, C, Shallice, T, Macaluso, E (2010) Item retrieval and competition in noun and verb generation: an FMRI study. J Cogn Neurosci, 22:1140-1157.

D'Esposito, M, Deouell, LY, Gazzaley, A (2003) Alterations in the BOLD fMRI signal with ageing and disease: a challenge for neuroimaging. Nat Rev Neurosci, 4:863-872.

Damoiseaux, JS, Rombouts, SA, Barkhof, F, Scheltens, P, Stam, CJ, Smith, SM, Beckmann, CF (2006) Consistent resting-state networks across healthy subjects. Proc Natl Acad Sci U S A, 103:13848-13853.

Dehaene, S, Cohen, L (2011) The unique role of the visual word form area in reading. Trends Cogn Sci, 15:254262.

Di Martino, A, Yan, CG, Li, Q, Denio, E, Castellanos, FX, Alaerts, K, Anderson, JS, Assaf, M, Bookheimer, SY, Dapretto, M, Deen, B, Delmonte, S, Dinstein, I, Ertl-Wagner, B, Fair, DA, Gallagher, L, Kennedy, DP, Keown, CL, Keysers, C, Lainhart, JE, Lord, C, Luna, B, Menon, V, Minshew, NJ, Monk, CS, Mueller, S, Muller, RA, Nebel, MB, Nigg, JT, O'Hearn, K, Pelphrey, KA, Peltier, SJ, Rudie, JD, Sunaert, S, Thioux, M, Tyszka, JM, Uddin, LQ, Verhoeven, JS, Wenderoth, N, Wiggins, JL, Mostofsky, SH, Milham, MP (2013) The autism brain imaging data exchange: towards a large-scale evaluation of the intrinsic brain architecture in autism. Mol Psychiatry, 19:659-667.

Fair, DA, Bathula, D, Nikolas, MA, Nigg, JT (2012) Distinct neuropsychological subgroups in typically developing youth inform heterogeneity in children with ADHD. Proc Natl Acad Sci U S A, 109:6769-6774.

Filippini, N, Maclntosh, BJ, Hough, MG, Goodwin, GM, Frisoni, GB, Smith, SM, Matthews, PM, Beckmann, CF, Mackay, CE (2009) Distinct patterns of brain activity in young carriers of the APOE-epsilon4 allele. Proc Natl Acad Sci U S A, 106:7209-7214. 
Fox, MD, Snyder, AZ, Vincent, JL, Corbetta, M, Van Essen, DC, Raichle, ME (2005) The human brain is intrinsically organized into dynamic, anticorrelated functional networks. Proc Natl Acad Sci U S A, 102:9673-9678.

Friston, K, Price, CJ (2003) Degeneracy and redundancy in cognitive anatomy. Trends in Cognitive Sciences, 7:151-152.

Gates, KM, Molenaar, PC, lyer, SP, Nigg, JT, Fair, DA (2014) Organizing heterogeneous samples using community detection of GIMME-derived resting state functional networks. PLoS One, 9:e91322.

Gorno-Tempini, ML, Dronkers, NF, Rankin, KP, Ogar, JM, Phengrasamy, L, Rosen, HJ, Johnson, JK, Weiner, MW, Miller, BL (2004) Cognition and anatomy in three variants of primary progressive aphasia. Ann Neurol, 55:335-346.

Grezes, J, Decety, J (2001) Functional anatomy of execution, mental simulation, observation, and verb generation of actions: a meta-analysis. Hum Brain Mapp, 12:1-19.

Handwerker, DA, Ollinger, JM, D'Esposito, M (2004) Variation of BOLD hemodynamic responses across subjects and brain regions and their effects on statistical analyses. Neurolmage, 21:1639-1651.

Holland, SK, Plante, E, Weber Byars, A, Strawsburg, RH, Schmithorst, VJ, Ball, WSJ (2001) Normal fMRI brain activation patterns in children performing a verb generation task. Neuroimage, 14:837-843.

Holmes, AP, Friston, K (1998) Generalisability, random effects and populaiton inference. Neurolmage, 7:S754.

Horowitz-Kraus, T, Vannest, JJ, Gozdas, E, Holland, SK (2014) Greater Utilization of Neural-Circuits Related to Executive Functions is Associated with Better Reading: A Longitudinal fMRI Study Using the Verb Generation Task. Front Hum Neurosci, 8:447.

Hyvarinen, A (1999) Fast and robust fixed-point algorithms for independent component analysis. IEEE Trans Neural Netw, 10:626-634.

Jafri, MJ, Pearlson, GD, Stevens, M, Calhoun, VD (2008) A method for functional network connectivity among spatially independent resting-state components in schizophrenia. Neuroimage, 39:1666-1681.

Jobard, G, Vigneau, M, Simon, G, Tzourio-Mazoyer, N (2011) The weight of skill: Interindividual variability of reading related brain activation patterns in fluent readers. Journal of Neurolinguistics, 24:113-132.

Karunanayaka, P, Schmithorst, VJ, Vannest, J, Szaflarski, JP, Plante, E, Holland, SK (2010) A group independent component analysis of covert verb generation in children: a functional magnetic resonance imaging study. Neuroimage, 51:472-487.

Karunanayaka, P, Schmithorst, VJ, Vannest, J, Szaflarski, JP, Plante, E, Holland, SK (2011) A linear structural equation model for covert verb generation based on independent component analysis of FMRI data from children and adolescents. Front Syst Neurosci, 5:29.

Kherif, $F$ (2003) Group analysis in functional neuroimaging: selecting subjects using similarity measures. Neurolmage, 20:2197-2208.

Kherif, F, Josse, G, Seghier, ML, Price, CJ (2009) The main sources of intersubject variability in neuronal activation for reading aloud. J Cogn Neurosci, 21:654-668.

Leiguarda, RC, Marsden, CD (2000) Limb apraxias: higher-order disorders of sensorimotor integration. Brain, 123:860-879.

Martin, RC, Cheng, Y (2006) Selection demands versus association strength in the verb generation task. Psychon Bull Rev, 13:396-401. 
Mazoyer, B, Zago, L, Jobard, G, Crivello, F, Joliot, M, Perchey, G, Mellet, E, Petit, L, Tzourio-Mazoyer, N (2014) Gaussian mixture modeling of hemispheric lateralization for language in a large sample of healthy individuals balanced for handedness. PLoS One, 9:e101165.

McKeown, MJ, Jung, TP, Makeig, S, Brown, G, Kindermann, SS, Lee, TW, Sejnowski, TJ (1998) Spatially independent activity patterns in functional MRI data during the stroop color-naming task. Proc Natl Acad Sci U S A, 95:803-810.

Miller, MB, Donovan, CL, Bennett, CM, Aminoff, EM, Mayer, RE (2012) Individual differences in cognitive style and strategy predict similarities in the patterns of brain activity between individuals. Neuroimage, 59:8393.

Miller, MB, Van Horn, JD (2007) Individual variability in brain activations associated with episodic retrieval: a role for large-scale databases. Int J Psychophysiol, 63:205-213.

Minka, T (2000) Automatic choice of dimensionality for PCA. MIT Media Lab Vision and Modelling Group, Technical Report 514, udd

Newman, ME (2006) Modularity and community structure in networks. Proc Natl Acad Sci U S A, 103:8577-8582.

Noppeney, U, Friston, KJ, Price, CJ (2004) Degenerate neuronal systems sustaining cognitive functions. J Anat, 205:433-442.

Noppeney, U, Penny, WD, Price, CJ, Flandin, G, Friston, KJ (2006) Identification of degenerate neuronal systems based on intersubject variability. Neuroimage, 30:885-890.

Persson, J, Sylvester, CY, Nelson, JK, Welsh, KM, Jonides, J, Reuter-Lorenz, PA (2004) Selection requirements during verb generation: differential recruitment in older and younger adults. Neuroimage, 23:1382-1390.

Picard, N, Strick, PL (2001) Imaging the premotor areas. Curr Opin Neurobiol, 11:663-672.

Poldrack, RA (2006) Can cognitive processes be inferred from neuroimaging data? Trends Cogn Sci, 10:59-63.

Price, CJ, Friston, KJ (2002) Degeneracy and cognitive anatomy. Trends Cogn Sci, 6:416-421.

Pruim, RH, Mennes, M, van Rooij, D, Llera, A, Buitelaar, JK, Beckmann, CF (2015) ICA-AROMA: A robust ICAbased strategy for removing motion artifacts from fMRI data. Neuroimage, 112:267-277.

Rombouts, SA, Damoiseaux, JS, Goekoop, R, Barkhof, F, Scheltens, P, Smith, SM, Beckmann, CF (2009) Model-free group analysis shows altered BOLD FMRI networks in dementia. Hum Brain Mapp, 30:256266.

Rombouts, SA, Goekoop, R, Stam, CJ, Barkhof, F, Scheltens, P (2005) Delayed rather than decreased BOLD response as a marker for early Alzheimer's disease. Neuroimage, 26:1078-1085.

Salimi-Khorshidi, G, Douaud, G, Beckmann, CF, Glasser, MF, Griffanti, L, Smith, SM (2014) Automatic denoising of functional MRI data: combining independent component analysis and hierarchical fusion of classifiers. Neuroimage, 90:449-468.

Schippers, MB, Renken, R, Keysers, C (2011) The effect of intra- and inter-subject variability of hemodynamic responses on group level Granger causality analyses. Neurolmage, 57:22-36.

Seghier, ML, Lee, HL, Schofield, T, Ellis, CL, Price, CJ (2008) Inter-subject variability in the use of two different neuronal networks for reading aloud familiar words. Neuroimage, 42:1226-1236.

Smith, SM, Fox, PT, Miller, KL, Glahn, DC, Fox, PM, Mackay, CE, Filippini, N, Watkins, KE, Toro, R, Laird, AR, Beckmann, CF (2009) Correspondence of the brain's functional architecture during activation and rest. Proc Natl Acad Sci U S A, 106:13040-13045.

Smith, SM, Jenkinson, M, Woolrich, MW, Beckmann, CF, Behrens, TE, Johansen-Berg, H, Bannister, PR, De Luca, M, Drobnjak, I, Flitney, DE, Niazy, RK, Saunders, J, Vickers, J, Zhang, Y, De Stefano, N, Brady, JM, 
Matthews, PM (2004) Advances in functional and structural MR image analysis and implementation as FSL. Neuroimage, 23 Suppl 1:S208-19.

Smith, SM, Nichols, TE, Vidaurre, D, Winkler, AM, Behrens, TE, Glasser, MF, Ugurbil, K, Barch, DM, Van Essen, DC, Miller, KL (2015) A positive-negative mode of population covariation links brain connectivity, demographics and behavior. Nat Neurosci, 18:1565-1567.

Tavor, I, Parker Jones, O, Mars, RB, Smith, SM, Behrens, TE, Jbabdi, S (2016) Task-free MRI predicts individual differences in brain activity during task performance. Science, 352:216-220.

Thiebaut de Schotten, M, Dell'Acqua, F, Forkel, SJ, Simmons, A, Vergani, F, Murphy, DG, Catani, M (2011) A lateralized brain network for visuospatial attention. Nat Neurosci, 14:1245-1246.

Thompson-Schill, SL, D'Esposito, M, Aguirre, GK, Farah, MJ (1997) Role of left inferior prefrontal cortex in retrieval of semantic knowledge: a reevaluation. Proc Natl Acad Sci U S A, 94:14792-14797.

Thompson-Schill, SL, D'Esposito, M, Kan, IP (1999) Effects of repetition and competition on activity in left prefrontal cortex during word generation. Neuron, 23:513-522.

Woolrich, MW, Behrens, TE, Beckmann, CF, Jenkinson, M, Smith, SM (2004) Multilevel linear modelling for FMRI group analysis using Bayesian inference. Neuroimage, 21:1732-1747.

Woolrich, MW, Jbabdi, S, Patenaude, B, Chappell, M, Makni, S, Behrens, T, Beckmann, C, Jenkinson, M, Smith, SM (2009) Bayesian analysis of neuroimaging data in FSL. Neuroimage, 45:S173-86.

Worsley, KJ, Marrett, S, Neelin, P, Vandal, AC, Friston, KJ, Evans, AC (1996) A unified statistical approach for determining significant signals in images of cerebral activation. Hum Brain Mapp, 4:58-73. 


\section{Tables}

\begin{tabular}{|c|c|c|c|c|c|}
\hline \multicolumn{6}{|c|}{ Subgroup 1: $N=6 ; M=4 F=2 ;$ median Age $=27 ; 6 \mathrm{RH} 0 \mathrm{LH}$} \\
\hline $\begin{array}{l}\text { Subgrou } \\
p\end{array}$ & Subj ID & Age & Sex & Annett score & handedness \\
\hline 1 & 4 & 46 & M & 11 & $\mathrm{RH}$ \\
\hline 1 & 6 & 27 & M & $\mathrm{N} / \mathrm{A}$ & $\mathrm{RH}$ \\
\hline 1 & 9 & 25 & $\mathrm{~F}$ & $\mathrm{~N} / \mathrm{A}$ & $\mathrm{RH}$ \\
\hline 1 & 15 & 22 & M & $\mathrm{N} / \mathrm{A}$ & $\mathrm{RH}$ \\
\hline 1 & 16 & 28 & $\mathrm{~F}$ & 24 & $\mathrm{RH}$ \\
\hline 1 & 17 & 27 & M & $\mathrm{N} / \mathrm{A}$ & $\mathrm{RH}$ \\
\hline \multicolumn{6}{|c|}{ Subgroup 2: $\mathrm{N}=22 ; \mathrm{M}=10 \mathrm{~F}=12 ;$ median Age $=31 ; 15 \mathrm{RH} 7 \mathrm{LH}$} \\
\hline $\begin{array}{l}\text { Subgrou } \\
p\end{array}$ & Subj ID & Age & Sex & Annett score & handedness \\
\hline 2 & 3 & 38 & $\mathrm{~F}$ & $\mathrm{~N} / \mathrm{A}$ & $\mathrm{RH}$ \\
\hline 2 & 5 & 32 & M & 17 & $\mathrm{RH}$ \\
\hline 2 & 11 & 28 & $\mathrm{M}$ & 22 & $\mathrm{RH}$ \\
\hline 2 & 12 & 47 & $\mathrm{~F}$ & 16 & $\mathrm{RH}$ \\
\hline 2 & 13 & 23 & $\mathrm{~F}$ & 13 & $\mathrm{RH}$ \\
\hline 2 & 14 & 30 & $F$ & $\mathrm{~N} / \mathrm{A}$ & $\mathrm{RH}$ \\
\hline 2 & 19 & 29 & $\mathrm{~F}$ & 18 & $\mathrm{RH}$ \\
\hline 2 & 20 & 21 & $M$ & 20 & $\mathrm{RH}$ \\
\hline 2 & 21 & 30 & $F$ & $\mathrm{~N} / \mathrm{A}$ & $\mathrm{RH}$ \\
\hline 2 & 23 & 38 & $\mathrm{~F}$ & 20 & $\mathrm{RH}$ \\
\hline 2 & 24 & 24 & $M$ & 20 & $\mathrm{RH}$ \\
\hline 2 & 25 & 21 & $\mathrm{~F}$ & 20 & $\mathrm{RH}$ \\
\hline 2 & 26 & 38 & $\mathrm{~F}$ & 24 & $\mathrm{RH}$ \\
\hline 2 & 27 & 20 & $M$ & 16 & $\mathrm{RH}$ \\
\hline 2 & 29 & 27 & $M$ & 18 & $\mathrm{RH}$ \\
\hline 2 & 30 & 46 & $M$ & -22 & $\mathrm{LH}$ \\
\hline 2 & 31 & 39 & $M$ & -24 & $\mathrm{LH}$ \\
\hline 2 & 33 & 34 & $M$ & -13 & $\mathrm{LH}$ \\
\hline 2 & 36 & 39 & $F$ & $\mathrm{~N} / \mathrm{A}$ & $\mathrm{LH}$ \\
\hline
\end{tabular}




\begin{tabular}{|c|c|c|c|c|c|}
\hline 2 & 39 & 21 & $\mathrm{~F}$ & -19 & LH \\
\hline 2 & 43 & 36 & $\mathrm{~F}$ & N/A & LH \\
\hline 2 & 44 & 40 & M & -20 & LH \\
\hline \multicolumn{6}{|c|}{ Subgroup 3: N=17; M=8 F=9; median Age $=31 ; 9 \mathrm{RH} 8 \mathrm{LH}$} \\
\hline $\begin{array}{l}\text { Subgrou } \\
\text { p }\end{array}$ & Subj ID & Age & Sex & Annett score & handedness \\
\hline 3 & 1 & 23 & M & 14 & $\mathrm{RH}$ \\
\hline 3 & 2 & 28 & M & 16 & $\mathrm{RH}$ \\
\hline 3 & 7 & 29 & M & 20 & $\mathrm{RH}$ \\
\hline 3 & 8 & 34 & $\mathrm{~F}$ & 24 & $\mathrm{RH}$ \\
\hline 3 & 10 & 39 & $\mathrm{~F}$ & 24 & RH \\
\hline 3 & 18 & 24 & M & 15 & $\mathrm{RH}$ \\
\hline 3 & 22 & 27 & M & 23 & $\mathrm{RH}$ \\
\hline 3 & 28 & 24 & $\mathrm{~F}$ & 20 & $\mathrm{RH}$ \\
\hline 3 & 32 & 31 & $\mathrm{~F}$ & -20 & LH \\
\hline 3 & 34 & 25 & $\mathrm{~F}$ & -21 & LH \\
\hline 3 & 35 & 31 & $\mathrm{~F}$ & -8 & LH \\
\hline 3 & 37 & 32 & $F$ & -14 & LH \\
\hline 3 & 38 & 47 & M & -8 & LH \\
\hline 3 & 40 & 28 & M & 18 & $\mathrm{RH}$ \\
\hline 3 & 41 & 24 & $\mathrm{~F}$ & -19 & LH \\
\hline 3 & 42 & 40 & $\mathrm{~F}$ & N/A & LH \\
\hline 3 & 45 & 34 & M & -20 & LH \\
\hline
\end{tabular}

Table 1: Participants' demographics in the different subgroups detected by our method. F=female; M=Male, $\mathrm{RH}=$ right-handed; $\mathrm{LH}=$ left-handed. As reported in the text (section 3.3) no significant difference in terms of age, sex and handedness was found across subgroups. 


\section{Supplementary Figures}

\section{Excluded ICA components}

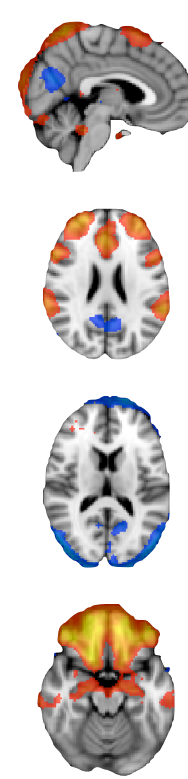

IC10: Mostly located outside of the brain (potential motion artifact and/ or sagittal sinus veins)

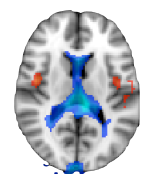

IC14: CSF fluctuations (cardiac pulsation)

IC11: Highly expressed in only one subject

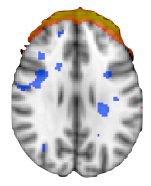

IC15: Motion artifact

IC12: Motion artifact

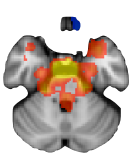

IC16: Cardiac pulsation

IC13: ssEPI susceptibility artifact

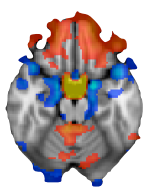

IC17: Unknown, possibly related to susceptibility artifacts and cardiac pulsation

Fig. S1: These ICA components, ordered by decreasing explained variance, were excluded by further analyses since they reflect sources of noise, or are highly expressed only in one subject (IC11). See also Salimi-Khorshidi et. al 2014 (reference in the main text) for a similar visualization and interpretation of the noise components
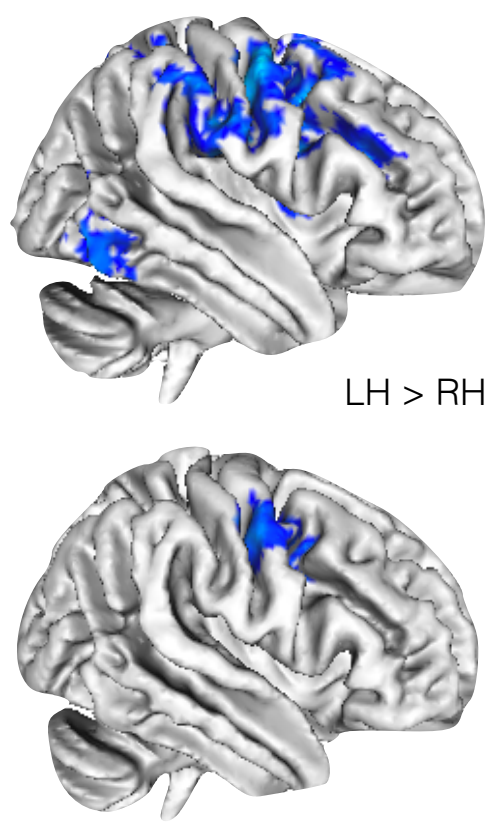

Annett: negative correlation

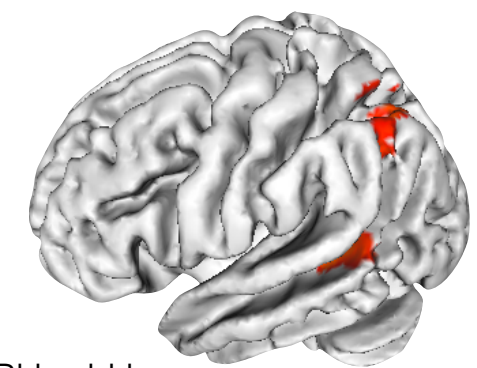

$\mathrm{RH}>\mathrm{LH}$

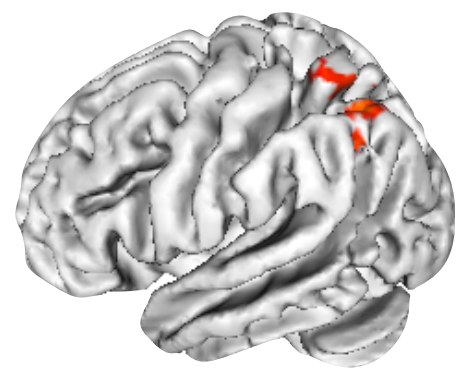

Annett: positive correlation

Fig. S2: Different brain activity during the VGEN task, evidenced by contrasting right-handed $(\mathrm{RH})$ with left-handed (LH) participants. A correlation with the Annett handedness scores (Annett, M 1970, full reference in the main text) on all the 35 (over 45) participants for which this score was available showed an effect in the regions where the contrast between $\mathrm{RH}$ and $\mathrm{LH}$ yielded the highest parameter estimates. 